\title{
Violacein anticancer activity is enhanced under hypoxia
}

\author{
SAEED M. HASHIMI ${ }^{1,2}$, TIEFENG XU ${ }^{3}$ and MING Q. WEI ${ }^{1}$ \\ ${ }^{1}$ Division of Molecular and Gene Therapies, Griffith Health Institute, School of Medical Science, Griffith University; \\ ${ }^{2}$ Molecular Basis for Disease, Griffith Health Institute, School of Dentistry and Oral Health, Griffith University, \\ Gold Coast, Qld 4215, Australia; ${ }^{3}$ Cancer Institute of Hainan Medical College, \\ Affiliated Hospital of Hainan Medical College, Haikou, Hainan 570102, P.R. China
}

Received November 28, 2014; Accepted December 29, 2014

DOI: $10.3892 /$ or.2015.3781

\begin{abstract}
Current cancer treatments of solid tumours such as chemotherapy and radiotherapy, have yet to produce effective therapeutic results due to non-specific targeting. This has led to many complications, such as toxicities in cancer patients. The ability of natural compounds in inducing programmed cell death (apoptosis), a process dysregulated in cancer cells, has been extensively studied in recent studies. This study assessed the anti-proliferative activity of violacein in a number of human cancer cell lines under normal and hypoxic conditions. Furthermore, we investigated its effects in a tumour-bearing subcutaneous mouse model. We also examined the ability of a tumour-targeting Salmonella strain to produce violacein for local delivery within the tumour microenvironment. The results showed that hypoxia significantly increased the cytotoxic effects of violacein. The most significant reduction in the $\mathrm{IC}_{50}$ was in the HT29 (12.6-fold) and HCT116 (4.8-fold) colon cancer cell lines, HN5 head and neck squamous carcinoma cell line (6.5-fold), and MCF-7 breast ductal carcinoma cell line (4-fold). Among the cell lines tested for active caspase-3/7 activity, violacein only increased caspase-3/7 activity in the A549 non-small lung cancer cell line. In vivo efficacy of violacein showed that HN5 tumour-bearing mice had regressed tumours during the treatment period and survival increased. The results also showed that transfer of the violacein biosynthetic cluster into the oncolytic strain VNP20009 of Salmonella resulted in the production of active violacein, suggesting targeted delivery of violacein by VNP20009. Taken together, our study has shown that hypoxia synergises the effects of violacein and the results from the in vivo administration of violacein require further investigation of violacein as an anticancer chemotherapeutic.
\end{abstract}

Correspondence to: Professor Ming Q. Wei, Division of Molecular and Gene Therapies, School of Medical Science, Griffith University, Gold Coast, Qld 4222, Australia

E-mail:m.wei@griffith.edu.au

Abbreviations: $\mathrm{IC}_{50}, 50 \%$ inhibitory concentration; MTT, 3-(4,5dimethylthiazol-2-yl)-2,5-diphenyltetrazolium bromide

Key words: violacein, chemotherapy, hypoxia, oncolytic Salmonella

\section{Introduction}

Cancer is one of the leading causes of mortality in humans. However, this problem is exasperated by the complex molecular nature of cancer and therefore requires a complex therapeutic strategy. Efforts have focused on the ability of natural compounds to induce programmed cell death and activate the relevant apoptotic cascades (1-4).

Violacein is a violet pigment that is produced from tryptophan by action of a cluster of five genes, designated as vioABCDE, naturally by the bacterium Chromobacterium violaceum $(5,6)$. It has significant biological and pharmacological activities, including antimicrobial, antiparasitary, anti-diarrhoeal, and antifungal activities (7-12). Violacein has been shown to possess anticancer properties (13-21) and has been overexpressed in E. coli K12 JM109 by cloning of the violacein gene cluster (22). Ahmetagic et al found that expression of the violacein gene cluster in the pPSX vector (JM109 E. coli K12 strain) and mutations in the promoter region produced hyper-producing strains of violacein (22).

Anticancer studies of violacein have shown efficacy in a number of cell lines of both neoplastic and haematological malignant origins. The most effective activity of violacein was against MOLT-4 leukaemia, NCI-H460 non-small-cell lung cancer and KM12 colon-cancer cell lines $\left(\mathrm{GI}_{50} \sim 30-60 \mathrm{nM}\right)(23)$. Furthermore, Saraiva et al found that uveal melanoma cell lines, 92.1 and OCM-1, were found to be sensitive to violacein $\left(\mathrm{GI}_{50} \sim 1.69-2.21 \mu \mathrm{M}\right)(21)$. Additionally, violacein was found to inhibit the growth and proliferation of colorectal cancer cell lines (16). The mechanism of action in HCT116 cells was identified as induction of apoptosis. Violacein was found to potentiate the effects of 5-fluorouracil in HCT116 cells. The in vivo efficacy of violacein has been assessed in intraperitoneal models of Ehrlich ascites tumour, which showed increased lifespan in tumour-bearing mice (13).

In terms of the mechanism of action of violacein, its role in the induction of apoptosis has been investigated and evidence suggests cell type-dependent activity (23). Findings of previous studies have indicated caspase-dependent apoptosis and production of reactive oxygen species as the mechanism of action $(14,16)$. The most comprehensive mechanistic study was undertaken by Ferreira et al and Kodach et al on leukaemia cells and colorectal cancer cells, respectively $(15,16)$. Violacein was found to activate TNF receptor 1-mediated apoptosis in HL60 
leukaemia cells (15). Furthermore, in HCT116 cells violacein was found to block cell cycle at G1, upregulate the expression of p53, p27 and p21 levels and decrease cyclin D1 expression. Most significantly, violacein was found to inhibit the phosphorylation of Akt, a serine-threonine-specific protein kinase involved in the proliferation and survival of many cancer types (16).

This study aimed to compare the cytotoxic effects of violacein in a number of cancer cells under normaxic and hypoxic conditions. Furthermore, we investigated the in vivo effect of violacein in subcutaneous tumour models. The results showed that hypoxia significantly potentiated the cytotoxic effects of violacein. Violacein was also found to cause tumour regression and have an increased lifespan in tumour-bearing mice. The results suggest additional investigations into the potential antitumour capacity of violacein.

\section{Materials and methods}

Mammalian cell culture conditions. Human cancer cell lines, A549 (lung), PC3 (prostate), HCT116 (colon), HT29 (colon), MCF-7 (breast), A431 (melanoma), HN5 (head and neck), and HeLa (cervix), were obtained from the American Type Culture Collection. The cell lines were cultured in Dulbecco's modified essential medium (Invitrogen-Life Technologies, Carlsbad, CA, USA) supplemented with 10\% heat-inactivated fetal bovine serum (FBS), $110 \mathrm{mg} / \mathrm{l}$ sodium pyruvate, $25 \mathrm{mM}$ HEPES, $100 \mathrm{U} / \mathrm{ml}$ of penicillin and $100 \mu \mathrm{g} / \mathrm{ml}$ of streptomycin solution, in an atmosphere of $5 \% \mathrm{CO}_{2}$ and $95 \%$ air at $37^{\circ} \mathrm{C}$. Hypoxic conditions were achieved by placing culture plates in a GasPak EZ pouch (BD) and incubating at $37^{\circ} \mathrm{C}$.

Expression and extraction of violacein. Violacein was expressed in the recombinant $E$. coli strain JM109 containing the violacein expression vector pBSX-vio-opv. This strain was kindly provided by Professor John Pemberton, The University of Queensland, Australia. The recombinant violacein expression vector $\mathrm{pBSX}$-vio-opv was also transformed into the tumour-targeting Salmonella typhimurium strain, VPN20009 (24). Rosenberg et al, showed that VPN20009 is able to colonise the tumour microenvironment 10,000-fold higher than that in liver in mice (24). The violacein-producing strains were cultured on PYE agar for $48 \mathrm{~h}$ until dark purple colonies were visible, indicating the production of violacein (22). The colonies were subsequently scraped from the culture plates and resuspended in water and centrifuged at $14,000 \mathrm{x}$ g. After centrifugation, the cells were resuspended in ethanol to extract the violacein. After centrifugation at $14,000 \mathrm{x} g$ the supernatant, containing the violacein, was collected and stored at $4^{\circ} \mathrm{C}$ until further use. The concentration of the extracted violacein was determined according to the methods of Duràn et al (25).

In vitro efficacy of violacein. Cytotoxicity of violacein in all the cell lines was studied by using 3-(4,5-dimethylthiazol2-yl)-2,5-diphenyltetrazolium bromide (MTT) assays (Invitrogen). Cells were seeded at a density of $1 \times 10^{4}$ cells per well in a 96-well plate. After seeding, the cells were exposed to violacein treatment for another $24 \mathrm{~h}$, and then MTT reagent was added following the manufacturer's instructions. The absorbance was read at $570 \mathrm{~nm}$ and $\mathrm{IC}_{50}$ values were calculated by Prism 5 (GraphPad Software).
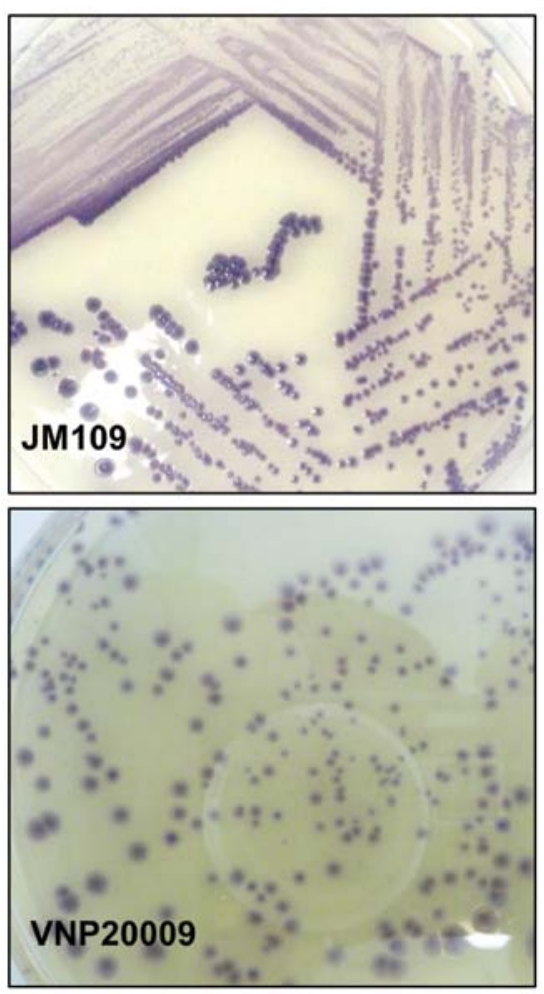

Figure 1.Production of violacein in E.coliJM109and Salmonella.typhimurium (S. typhimurium) VNP90001. The violacein biosynthetic cluster in the pBSX-vio-opv vector was transferred into E. coli JM109 and S. typhimurium VNP90001. Violacein production was induced by culturing the bacteria on PYE media. Purple pigmentation is indicative of violacein production.

Apoptosis assays. Cancer cell lines were seeded at a density of $1 \times 10^{4}$ cells per well in white $\mu$ Clear 96-well plates (Greiner) and exposed to violacein for $24 \mathrm{~h}$. Caspase-3 activity was evaluated using Caspase-Glo 3/7 assay systems (Promega, Madison, WI, USA) according to the manufacturer's instructions. Luciferase intensity was measured by POLARstar Omega at $24 \mathrm{~h}$ post-violacein addition.

Mouse xenograft models and violacein antitumour evaluation. Experiments involving animals were approved by the Griffith University animal ethics committee (AEC No. MSC/01/08). To establish mouse models, $5 \times 10^{6} \mathrm{HN} 5$ cells in PBS $(200 \mu \mathrm{l})$ were subcutaneously injected into the right flank of female BALB/c nude mice. When tumours reached a size of $320 \mathrm{~mm}^{3}$, the mice (aged 8 weeks and weighing 16-18 g) were randomly divided into two groups (6 mice per group): the treatment group, which was administered $0.7 \mathrm{mg} / \mathrm{kg}$ body weight violacein, while an equal amount of injection solution without violacein was administered to the control group. Treatments were delivered by intraperitoneal injection. Tumour volume was measured using a calliper and calculated using the formula: $0.5\left(1 \mathrm{x} \mathrm{w}^{2}\right)$. When the tumour volume reached $1 \mathrm{~cm}^{3}$, mice were sacrificed by cervical dislocation.

Statistical analysis. Experiments were repeated at least three times to obtain statistically significant data. The results are presented as means with standard error of mean. The Student's $\mathrm{t}$-test was used to analyse the data and $\mathrm{p}<0.05$ was considered statistically significant. 

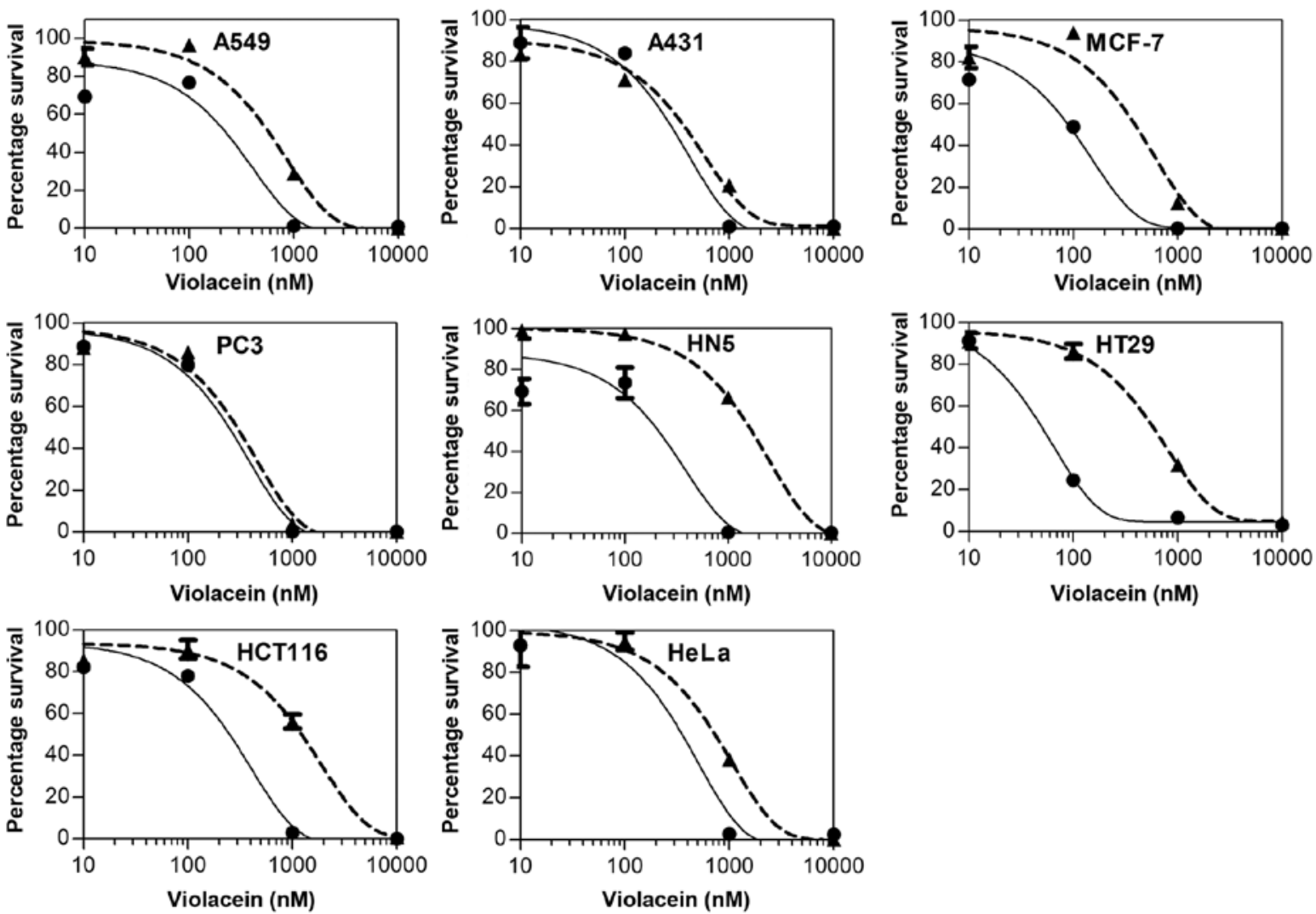

Figure 2. Violacein anti-proliferative effect on cancer cells. MTT assays were performed on several cancer cell lines to ascertain the anticancer effects of violacein. The solid line is the dose response in hypoxic conditions, while the dotted line is under normal cell culture conditions. Violacein was shown to be more effective under hypoxia in most cell lines.

\section{Results}

Production of violacein. Recombinant E. coli and Salmonella typhimurium strain VPN20009 containing the violacein biosynthetic clusters were cultured on PYE agar. After $48 \mathrm{~h}$ intense purple-coloured colonies were visible in the two strains (Fig. 1). The intense purple colourisation in the VPN20009 plates showed that it is capable of producing violacein from the pBSX-vio-opv expression vector. Following extraction and purification, the violacein content was measured by spectrophotometry at $570 \mathrm{~nm}$. From a batch of 20 plates, $5 \mathrm{ml}$ of $3 \mathrm{mM}$ violacein was extracted in ethanol. This extract was subsequently used in the cytotoxic assays and in the xenograft tumour models.

Violacein is a potent inhibitor of cancer cell proliferation. To examine the effects of violacein on cells MTT proliferation assays were used. MTT was converted to formazan by living cells and was detected by spectrophotometric quantification. Violacein was tested at a concentration range of $0-10 \mu \mathrm{M}$ to yield a dose response for subsequent $\mathrm{IC}_{50}$ calculation. The results showed that violacein was highly potent against A549 non-small cell lung cancer, A431 Melanoma, MCF-7 breast cancer, PC3 prostate cancer, HT29 colon cancer, and HeLa cervical cancer (Fig. 2). Furthermore, violacein was effective against HCT116 colon and HN5 head and neck cancer, although not as potent as against the other cell lines. The dose response curve was used to calculate $\mathrm{IC}_{50}$ concentrations for the various cell lines (Table I). Based on the $\mathrm{IC}_{50}$ values
Table I. Anticancer activity of violacein.

\begin{tabular}{llccc}
\hline & & \multicolumn{2}{c}{ Violacein $\mathrm{IC}_{50}(\mathrm{nM})$} & \\
\cline { 3 - 4 } Cell line & \multicolumn{1}{c}{$\begin{array}{c}\text { Tissue } \\
\text { origin }\end{array}$} & $\begin{array}{c}\text { Fold drop } \\
\mathrm{IC}_{50}\end{array}$ \\
\hline A549 & Lung & 285.5 & 601.8 & 2.1 \\
A431 & Skin & 288.2 & 412.6 & 1.4 \\
MCF-7 & Breast & $\mathbf{1 0 5 . 6}$ & 421 & 4 \\
PC3 & Prostate & 268.5 & 318.2 & 1.2 \\
HCT116 & Colon & $\mathbf{2 8 3 . 5}$ & 1348 & 4.8 \\
HT29 & Colon & $\mathbf{4 5 . 2}$ & 569.4 & 12.6 \\
HN5 & Head and neck & $\mathbf{2 6 8 . 4}$ & 1738 & 6.5 \\
HeLa & Cervix & 351.9 & 744 & 2.1 \\
\hline
\end{tabular}

Bold, cell lines with significantly lower $\mathrm{IC}_{50}$ under hypoxic conditions.

violacein was most effective against A431, MCF-7 and PC3 cancer cells, while HN5 and HCT116 were the least affected. A microscopic analysis of the treated cells revealed fewer and dead cells compared to the non-treated group (Fig. 3). Furthermore, the morphology of violacein-treated cells was round compared to a flat appearance of the control cells.

Hypoxia sensitises cancer cells to violacein. To determine the effects of violacein on cancer cells in hypoxic conditions, 


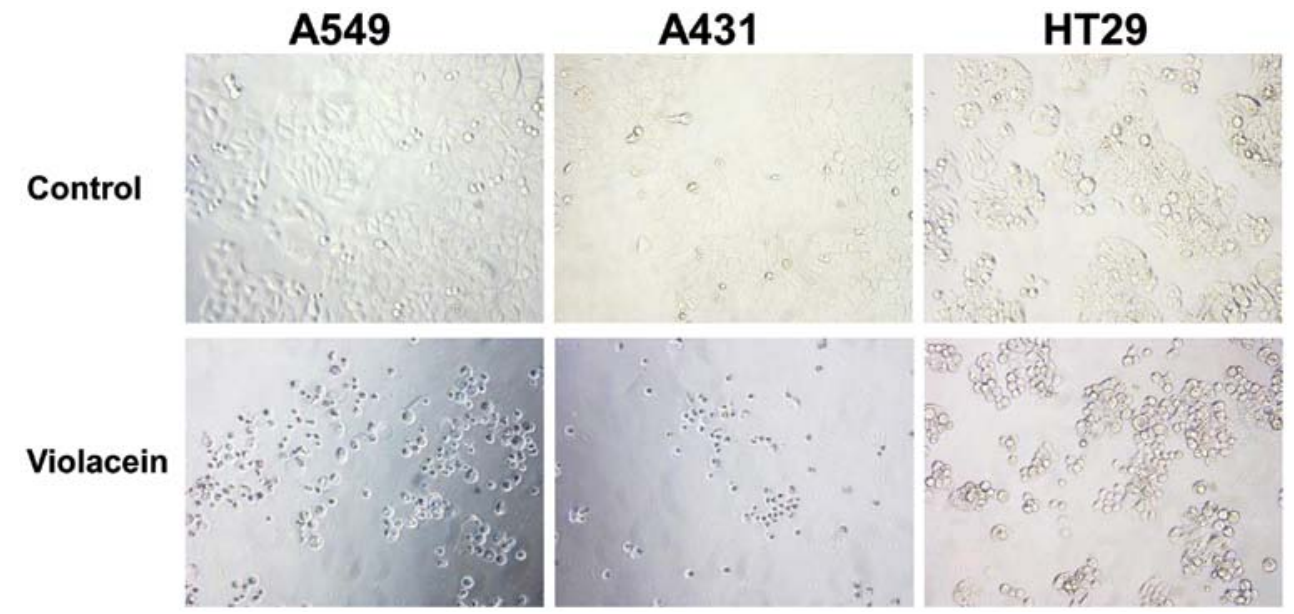

Figure 3. Microscopic analysis of cancer cells treated with violacein. Treated cells show a round morphology compared to non-treated cells. Violacein-treated cells are fewer in number and are detached from the culture plate surface.

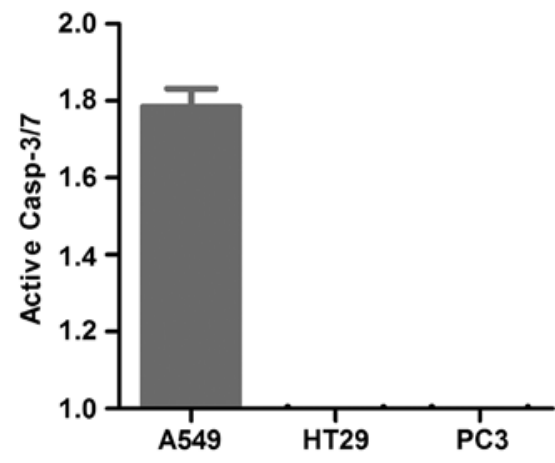

Figure 4. Caspase 3/7 activity in cancer cells induced by violacein. Active caspase-3/7 was only induced in A549 cells, while the remaining cells showed no change.

the cells were cultured in a GasPak EZ pouch (BD) at $37^{\circ} \mathrm{C}$. MTT proliferation assays showed that hypoxia sensitised all the cancer cells to violacein. The most significant effect was on MCF-7 breast ductal carcinoma, HCT116 and HT29 colon cancer, and HN5 head and neck squamous carcinoma cells (Fig. 2). Furthermore, a dose response curve was used to calculate the $\mathrm{IC}_{50}$ values for violacein under hypoxia and normoxia. It was found that HT26 was 12.6-fold more sensitive to violacein under hypoxia when compared to normoxic conditions (Table I). The $\mathrm{IC}_{50}$ values for PC3 prostate cancer and $\mathrm{A} 431$ melanoma cells were not significantly affected by hypoxia.

Violacein activates caspase-dependent apoptosis in A549 cells only. To determine whether violacein activates caspase dependent apoptosis, the cells were seeded in 96-well white walled plates, exposed to violacein and the caspase-3/7 activity was measured. Caspase-3/7 activity was induced in A549 non-small cell lung cancer cells only when treated with violacein (Fig. 4). The remaining cell lines tested had similar caspase-3/7 activity in the treated and non-treated cells.

In vivo activity of violacein. Having established the efficacy of violacein in the in vitro culture system we elucidated its activity in xenograft tumour models. Head and neck squamous
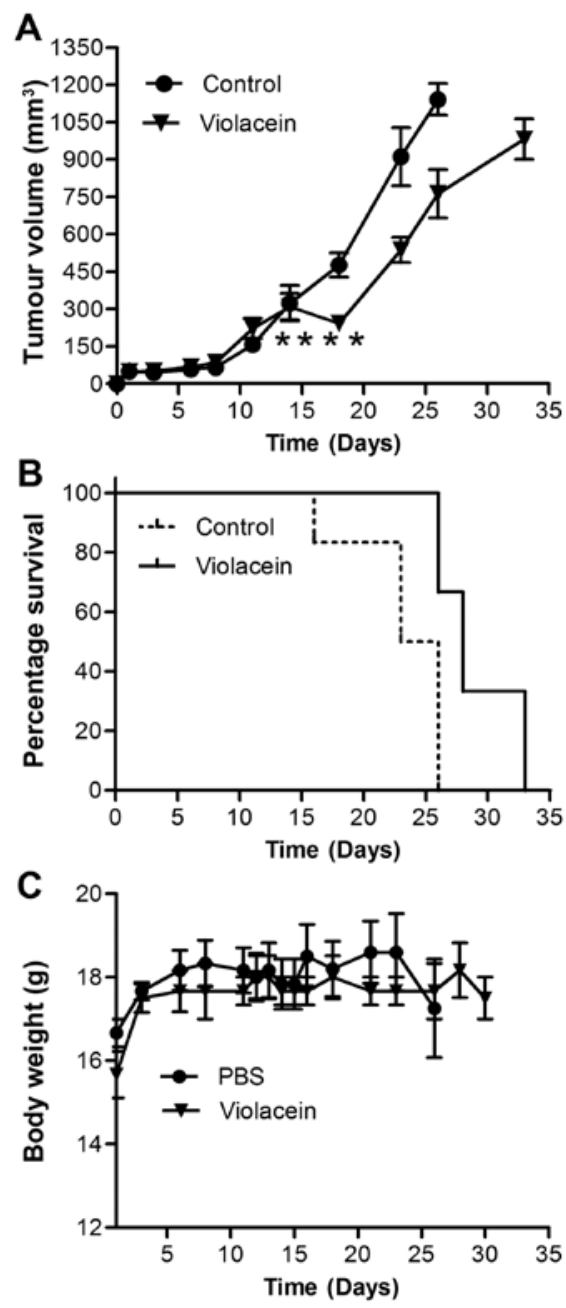

Figure 5. In vivo efficacy of violacein against mice tumour models. (A) The effect of violacein on tumour volume compared to control groups. (B) Survival of tumour-bearing mice in response to violacein or vehicle (Control). (C) Body weight of mice during the treatment period.

carcinoma subcutaneous xenograft models were established in $\mathrm{BALB} / \mathrm{c}$ nude mice and treated with daily doses of $0.7 \mathrm{mg} / \mathrm{kg}$ of body weight for 4 days. It was found that tumours treated with violacein regressed during the treatment period while 
the non-treated control tumours continued to grow (Fig. 5). Furthermore, violacein-treated tumours had a significantly slower growth rate compared to the control groups. It was also found that violacein extended the life span of animals during the treatment period, however, the mortality rate reached similar levels as that of the controls when treatment was terminated. Violacein administration did not affect the weight of the animals and treated animals were not any different to controls in terms of behaviour or phenotypic features.

\section{Discussion}

Cancer remains a devastating disease inflicting significant mortality rates to humans worldwide despite significant breakthroughs in its diagnosis and treatment. Natural compounds have been extensively studied for their biological activities and in particular their ability to induce programmed cell death or apoptosis in cancer cells (1-4). Understanding the mechanisms by which these drugs kill cancer cells provides the possibility of engineering novel drugs with improved anticancer activity.

Violacein produced by the bacterium Chromobacterium violaceum, a broad spectrum bioactive compound, has been shown to have anticancer activity (23). Our study focused on understanding the mechanisms of action of violacein in cancer cells.

Cell proliferation assays were used to determine the in vitro effects of violacein on different cancer cell lines. Our results indicated a broad range and cell-specific violacein-mediated cytotoxicity. Furthermore, active caspase-3/7 measurements showed that apoptosis was induced only in A549 cells, indicating a differential effect of violacein on inducing apoptosis. A number of other investigators have shown that violacein induces apoptosis in colorectal cancer cells (16), Ehrlich ascites tumour cells (13), and HL60 leukemia cells (15). Notably, Ferreira et al found that violacein only induced apoptosis in HL60 leukemia cells and was ineffective in other types of leukemia cells indicating a differential apoptosis-inducing activity (15). Furthermore, we found that the anti-proliferative activity of violacein increased when the cells were exposed to hypoxic conditions. In addition, the most significant effect was observed in HT29 (colon cancer) and HN5 (head and neck cancer) cells showing a 12.6- and 6.5-fold decrease, respectively, in $\mathrm{IC}_{50}$ from normoxic conditions. Of note, chemotherapeutic drugs that target proliferating cells are less effective in hypoxic conditions when cancer cells are quiescent (26). Furthermore, it has been well established that the quiescent cells, especially cancer stem cells, are involved in tumour progression and metastasis (27). This has limited the use of many chemotherapeutic, making them mostly palliative. The apparent increased in vitro activity of violacein in hypoxic conditions suggests a capacity in killing non-proliferative cells, a property with potential promise in combating chemoresistant cells.

Since violacein can be produced in E. coli carrying the recombinant plasmid pBSX-vio-opv, we determined the ability of the tumour-colonising Salmonella typhimurium strain VPN20009 to produce violacein from pBSX-vio-opv. Our results showed that this strain was capable of producing violacein when transformed with the pBSX-vio-opv plasmid. Furthermore, cytotoxicity assays showed that the violacein produced was effective in killing cancer cells. Combining the tumour-colonising property of Salmonella typhimurium strain VPN20009 with violacein expression offers a potential target delivery system for violacein to the tumour core (28). Future studies are needed to ascertain the efficacy of this genetically modified strain.

The efficacy of violacein was also assessed in vivo on xenograft tumour models of HN5 (head and neck cancer) cells in mice. Our results showed that violacein regressed tumour volume and increased survival. However, tumour volumes reached the same levels as those of the controls when treatment was stopped. Additional studies with a prolonged dose regime are likely to yield an improved response to violacein. The in vivo results are encouraging for future studies to employ the tumour-targeting ability of VNV20009 $(24,29)$ for the local production of violacein in the tumour microenvironment.

In conclusion, our findings confirm the anti-proliferative properties of violacein in a number of cancer cell lines and that this activity is increased in hypoxia-induced cells. Furthermore, violacein can be expressed in the antitumour Salmonella typhimurium strain VPN20009, a potentially promising strategy to deliver violacein locally to tumours. Our study further showed that violacein apoptotic activity is differential and in vivo violacein showed anti-proliferative activity against subcutaneous head and neck tumours. The findings of this study require further studies to ascertain the anticancer properties of violacein and its local delivery by genetically modified tumour-colonising bacteria harbouring the violacein biosynthetic cluster.

\section{Acknowledgements}

This study was supported by the Dr Jian Zhou Smart State fellowship from the Queensland government, and grants from the National Health and Medical Research Council and Cancer Council, Queensland to M.Q.W. The National Natural Science Foundation of China (grant no. 81160297) supported Tiefeng Xu. We would like to thank other members of the Wei Laboratory for their support and helpful comments. Special thanks is extended to Professor John Pemberton for providing the violacein producing E. coli strain (pBSX-vio-opv).

\section{References}

1. Tabata K, Yamazaki Y, Okada M, et al: Diarylheptanoids derived from Alpinia officinarum induce apoptosis, S-phase arrest and differentiation in human neuroblastoma cells. Anticancer Res 29: 4981-4988, 2009.

2. Uddin S, Khan AS and Al-Kuraya KS: Developing curcumin into a viable therapeutic for lymphoma. Expert Opin Investig Drugs 18: 57-67, 2009.

3. Wang TT, Schoene NW, Kim YS, Mizuno CS and Rimando AM: Differential effects of resveratrol and its naturally occurring methylether analogs on cell cycle and apoptosis in human androgen-responsive LNCaP cancer cells. Mol Nutr Food Res 54: 335-344, 2010.

4. Li C, Hashimi SM, Cao S, et al: The mechanisms of chansu in inducing efficient apoptosis in colon cancer cells. Evid Based Complement Alternat Med 2013: 849054, 2013.

5. August PR, Grossman TH, Minor C, et al: Sequence analysis and functional characterization of the violacein biosynthetic pathway from Chromobacterium violaceum. J Mol Microbiol Biotechnol 2: 513-519, 2000.

6. Pemberton JM, Vincent KM and Penfold RJ: Cloning and heterologous expression of the violacein biosynthesis gene cluster from Chromobacterium violaceum. Curr Microbiol 22: 355-358, 1991. 
7. Andrighetti-Fröhner CR, Antonio RV, Creczynski-Pasa TB, Barardi CR and Simões CM: Cytotoxicity and potential antiviral evaluation of violacein produced by Chromobacterium violaceum. Mem Inst Oswaldo Cruz 98: 843-848, 2003.

8. Antonisamy P and Ignacimuthu S: Immunomodulatory, analgesic and antipyretic effects of violacein isolated from Chromobacterium violaceum. Phytomedicine 17: 300-304, 2010.

9. Antonisamy P, Kannan P and Ignacimuthu S: Anti-diarrhoeal and ulcer-protective effects of violacein isolated from Chromobacterium violaceum in Wistar rats. Fundam Clin Pharmacol 23: 483-490, 2009.

10. Cazoto LL, Martins D, Ribeiro MG, Durán N and Nakazato G: Antibacterial activity of violacein against Staphylococcus aureus isolated from bovine mastitis. J Antibiot 64: 395-397, 2011.

11. Leon LL, Miranda CC, De Souza AO and Durán N: Antileishmanial activity of the violacein extracted from Chromobacterium violaceum. J Antimicrob Chemother 48: 449-450, 2001.

12. Lichstein HC and Van De Sand VF: The antibiotic activity of violacein, prodigiosin, and phthiocol. J Bacteriol 52: 145-146, 1946.

13. Bromberg N, Dreyfuss JL, Regatieri CV, et al: Growth inhibition and pro-apoptotic activity of violacein in Ehrlich ascites tumor. Chem Biol Interact 186: 43-52, 2010.

14. de Carvalho DD, Costa FT, Durán N and Haun M: Cytotoxic activity of violacein in human colon cancer cells. Toxicol In Vitro 20: 1514-1521, 2006.

15. Ferreira CV, Bos CL, Versteeg HH, Justo GZ, Durán N and Peppelenbosch MP: Molecular mechanism of violacein-mediated human leukemia cell death. Blood 104: 1459-1464, 2004.

16. Kodach LL, Bos CL, Durán N,Peppelenbosch MP, Ferreira CV and Hardwick JC: Violacein synergistically increases 5-fluorouracil cytotoxicity, induces apoptosis and inhibits Akt-mediated signal transduction in human colorectal cancer cells. Carcinogenesis 27 508-516, 2006.

17. Martins D, Frungillo L, Anazzetti MC, Melo PS and Durán N Antitumoral activity of L-ascorbic acid-poly- D,L-(lactide-coglycolide) nanoparticles containing violacein. Int J Nanomed 5: $77-85,2010$.

18. Melo PS, Justo GZ, de Azevedo MB, Durán N and Haun M: Violacein and its beta-cyclodextrin complexes induce apoptosis and differentiation in HL60 cells. Toxicology 186: 217-225, 2003.
19. Melo PS, Maria SS, Vidal BC, Haun M and Durán N: Violacein cytotoxicity and induction of apoptosis in V79 cells. In Vitro Cell Dev Biol Anim 36: 539-543, 2000.

20. Queiroz KC, Milani R, Ruela-de-Sousa RR, et al: Violacein induces death of resistant leukaemia cells via kinome reprogramming, endoplasmic reticulum stress and Golgi apparatus collapse. PLoS One 7: e45362, 2012.

21. Saraiva VS, Marshall JC, Cools-Lartigue J and Burnier MN Jr: Cytotoxic effects of violacein in human uveal melanoma cell lines. Melanoma Res 14: 421-424, 2004.

22. Ahmetagic A and Pemberton JM: Stable high level expression of the violacein indolocarbazole anti-tumour gene cluster and the Streptomyces lividans amyA gene in E. coli K12. Plasmid 63: 79-85, 2010.

23. Durán N, Justo GZ, Ferreira CV, Melo PS, Cordi L and Martins D: Violacein: properties and biological activities. Biotechnol Appl Biochem 48: 127-133, 2007.

24. Rosenberg SA, Spiess PJ and Kleiner DE: Antitumor effects in mice of the intravenous injection of attenuated Salmonella typhimurium. J Immunother 25: 218-225, 2002.

25. Durán N, Antonio RV, Haun M and Pilli RA: Biosynthesis of a trypanocide by Chromobacterium violaceum. World J Microbiol Biotechnol 10: 686-690, 1994.

26. Rohwer $\mathrm{N}$ and Cramer T: Hypoxia-mediated drug resistance: novel insights on the functional interaction of HIFs and cell death pathways. Drug Resist Updat 14: 191-201, 2011.

27. Tirino V, Desiderio V, Paino F, et al: Cancer stem cells in solid tumors: an overview and new approaches for their isolation and characterization. FASEB J 27: 13-24, 2013.

28. Hashimi SM, Yu S, Alqurashi N, Ipe DS and Wei MQ: Immunotoxin-mediated targeting of claudin- 4 inhibits the proliferation of cancer cells. Int J Oncol 42: 1911-1918, 2013.

29. Cunningham C and Nemunaitis J: A phase I trial of genetically modified Salmonella typhimurium expressing cytosine deaminase (TAPET-CD, VNP20029) administered by intratumoral injection in combination with 5-fluorocytosine for patients with advanced or metastatic cancer. Protocol no: CL-017. Version: April 9, 2001. Hum Gene Ther 12: 1594-1596, 2001. 